\title{
FEATURE RELEVANCE ASSESSMENT OF MULTISPECTRAL AIRBORNE LIDAR DATA FOR TREE SPECIES CLASSIFICATION
}

\author{
N. Amiri ${ }^{1,2 *}$ M. Heurich ${ }^{3}$, P. Krzystek ${ }^{1}$, A. K. Skidmore ${ }^{2,4}$ \\ ${ }^{1}$ Department of Geoinformatics, Munich University of Applied Sciences, Munich, Germany - (n.amiri, peter.krzystek)@hm.edu \\ ${ }^{2}$ Department of Natural Resources, Faculty of Geo-Information Science and Earth Observation (ITC), University of Twente, \\ Enschede, The Netherlands - (n.amiri, a.k.skidmore)@utwente.nl \\ ${ }^{3}$ Chair of Wildlife Ecology and Management, University of Freiburg, Freiburg, Germany - (marco.heurich)@wildlife.uni-freiburg.de \\ ${ }^{4}$ Department of Environmental Science, Macquarie University, NSW, 2106, Australia - (andrew.skidmore)@mq.edu.au
}

Commission III, WG III/5

KEY WORDS: Multispectral Lidar, 3D point clouds, Intensity, Tree Species Classification, Feature Analysis.

\begin{abstract}
:
The presented experiment investigates the potential of Multispectral Laser Scanning (MLS) point clouds for single tree species classification. The basic idea is to simulate a MLS sensor by combining two different Lidar sensors providing three different wavelngthes. The available data were acquired in the summer 2016 at the same date in a leaf-on condition with an average point density of 37 points $/ \mathrm{m}^{2}$. For the purpose of classification, we segmented the combined 3D point clouds consisiting of three different spectral channels into 3D clusters using Normalized Cut segmentation approach. Then, we extracted four group of features from the 3D point cloud space. Once a varity of features has been extracted, we applied forward stepwise feature selection in order to reduce the number of irrelevant or redundant features. For the classification, we used multinomial logestic regression with $L_{1}$ regularization. Our study is conducted using 586 ground measured single trees from 20 sample plots in the Bavarian Forest National Park, in Germany. Due to lack of reference data for some rare species, we focused on four classes of species. The results show an improvement between 4-10 pp for the tree species classification by using MLS data in comparison to a single wavelength based approach. A cross validated (15-fold) accuracy of 0.75 can be achieved when all feature sets from three different spectral channels are used. Our results cleary indicates that the use of MLS point clouds has great potential to improve detailed forest species mapping.
\end{abstract}

\section{INTRODUCTION}

Accurate assessment of tree species distribution of natural and commercial forests provides valuable information for forest management and planning purposes. Much efforts has been dedicated to single tree species classification approaches from remote sensing data to provide an effective means for forest inventory at large-scale. The single tree based approach has advantages over the area based one by providing accurate forest attributes for mixed and complex stands. However, errors in the single tree detection procedure can negatively affect the classification accuracy (Yao et al., 2012).

The derivation of numerous features from single wavelength ALS (Airborne Laser Scanning) point clouds has become standard in the remote sensing applications for tree species classification. Previous approaches showed that single wavelength ALS data at a wavelength of $1550 \mathrm{~nm}$ could be used to successfully classify coniferous and deciduous trees with an overall accuracy up to 0.9 (Yao et al., 2012). However, the classification accuracy drops down by 0.2 if a detailed tree species mapping is required. Recently, a number of studies have reported accuracy improvements by applying a set of Lidar waveform features. As an example, common ALS point cloud features such as the mean and standard deviation of pulse width within single laser beams have been mentioned to be amongst the essential variables when classifying broadleaved and coniferous trees from Lidar waveform is concerned (Reitberger et al., 2009). Furthermore, a study

\footnotetext{
*Corresponding author
}

of Hovi et al. (2016) focused on a systematic analysis for the discrimination potential of ALS point cloud features by analyzing the source behind the within-species variation.

On the other hand, features extracted from spectral imagery combined with ALS point clouds has also known as a common technique for tree species classification. Passive sensors by measuring the spectral response of emitted radiation by the sun and reflected by the canopy can provide useful information to classify tree species. However, the available techniques which combine aerial, multispectral or hyperspectral imageries with ALS point clouds for tree species classification operate only at a 2D grid level and discard 3D structural information. Studies that directly compared the use of ALS point clouds and spectral imagery often reported better results for the optical datasets (Fassnacht et al., 2016). Holmgren et al. (2008) classified three species in a boreal forest and achieved an overall accuracy of 0.96 when both multispectral and ALS data are combined. Furthermore, Lidarderived (e.g., Jakubowski et al. (2013); Jones et al. (2010)) or image-based (e.g., Waser et al. $(2010,2011)$ ) vegetation height features can be used to separate tree species. However, Ghosh et al. (2014) and Jones et al. (2010) mentioned in their studies that the classification results based on spectral information of hyperspectral data alone is often not successful in terms of notable improvement. Therefore, there are parameters that limit the effective operational use of the combined datasets (Packalén et al., 2009; Puttonen et al., 2010). The geometric registration due to various acquisition times or using dissimilar sensor types is one of the main challenging issue for combining two different datasets.

The recently developed Multispectral Laser Scanning (MLS) 
technique is becoming an interesting tool for precise forest mapping. Since it can provide not only a dense point cloud, but spectral information as well. There are some studies that have demonstrated the higher potential of MLS data for tree species classification (Lindberg et al., 2015; St-Onge and Budei, 2015; Yu et al., 2017). Lindberg et al. (2015) simulated MLS data by separate instruments and from various flights with a point density around 20 points $/ \mathrm{m}^{2}$ for characterization of tree species. St-Onge and Budei (2015) used intensity based features extracted from three spectral channels of Titan multispectral lidar system to classify broadleaved vs. needle-leaf trees with the classification error of $4.59 \%$ in Canada. Yu et al. (2017) with the same sensor achieved an overall accuracy of $85.6 \%$ for tree species classification in southern Finland using a combination of MLS point clouds and intensity based features. So far, the combination of various features from MLS point clouds has been examined but there is still potential to incorporate various structural and spectral information available in the point clouds for classification purposes. The main objectives of this study are (i) to estimate tree species classification accuracy using features extracted from MLS point cloud and (ii) to investigate the effect of feature selection step on the classification accuracy.

The remainder of this work is structured as follows: Section 2 focuses on the details of our approach. Section 3 shows the materials and experiment. Finally, the results are discussed with conclusions in Sections 4 and 5.

\section{METHOD}

The simulated MLS point cloud consisting of three different spectral channels (wavelengths) is segmented into $3 \mathrm{D}$ clusters representing single trees using the Normalized Cut algorithm. Note that we regard the segmentation as an external procedure in this study and we focus on the tree species classification step. Then, we perform the feature extraction in $3 \mathrm{D}$ point cloud space. In the following, we explain the extracted features, stepwise feature selection and classification steps in detail.

\subsection{MLS point cloud features}

MLS point clouds can provide a range of features related mainly to the 3D structure of single trees. The features derived from point clouds can be grouped into four main categories:

1. Geometric features. Axis length of paraboloid fitted to tree crown, percentiles of height distribution of points in tree cluster, percentage of points per height layer of tree, ratios of point counts by reflection type (Single, first, middle) for each spectral channel, totaling 26 features for each spectral channel.

2. Radiometric features. These features are referred to specific parameters which are generated by a waveform decomposition such as intensity. Histograms of reflection intensities and pulse widths inside the tree cluster, mean intensity of single and first reflections (Reitberger et al., 2009; Yao et al., 2012), in total 23 features for each spectral channel.

3. Bag-of-Words Model (BoM). We derived 8 geometric features from the local covariance matrix of each point as proposed in (Weinmann et al., 2013). Then, we constructed frequency histograms for each feature, using 2-14 bins, which gave rise to a simple Bag-of-Words model. This was done for 7 sizes of the spherical point neighborhood with radii
0.2-1.6 $\mathrm{m}$ used for computing the covariance matrix, resulting for each channel in 3304 features. The number of bins was determined empirically, to limit the generated feature count and to avoid overfitting.

4. Basic geometric properties such as crown polygon area and crown geometry.

\subsection{Feature selection}

The numerous extracted features raise the methodical problem that a large hyper-dimensional feature space faces a frequently spare number of reference samples (Fassnacht et al., 2016). Feature selection methods can be used to identify and remove irrelevant and redundant attributes from data that do not contribute to the accuracy of a classification model.

We apply forward stepwise selection (Hastie et al., 2001), where we start with a small number of features, and then proceed in an iterative fashion picking one additional feature in each round. A single iteration inspects every available feature candidate by adding it to the currently active feature set and obtaining an estimate of the classification error rate on the augmented data through cross-validation. The feature whose introduction into the active set yields the lowest error rate is incorporated into the result set, and the iteration continues. The process is terminated when the inclusion of additional feature ceases to decrease the classification error rate. It should be noted that this method requires significant computational effort due to the need to constantly retrain the classifier model as part of the cross-validation for accuracy assessment purposes.

\subsection{Classification}

We apply multinomial logistic regression with $L 1$ regularization to classify the single tree species based on the features extracted and discussed in the sec.2.1. Logistic regression models the probability distribution of the class label y as follow:

$$
p\left(y_{i}=k \mid x_{i} ; \Theta\right)=\exp \left(\theta_{k}^{T} x_{i}\right) /\left[1+\sum_{l}\left(\theta_{l}^{T} x_{i}\right)\right]
$$

where $x_{i} \in X, i=1, \ldots, N$ denotes $N$ feature vectors of training examples and $y_{i} \in 0,1$ their corresponding binary labels. Training the model amounts to maximizing the joint log-liklihood of the training examples in Eq. 2 with respect to the $>0$ term as:

$$
\min _{\Theta} \sum_{i=1}^{N}-\log p\left(y_{i} \mid x_{i} ; \Theta\right)+\beta\|\Theta\|_{1}
$$

where $\|\Theta\|_{1}$ is the regularization term, which promotes sparsity of the coefficient vector $\Theta$, resulting in many weights being exactly zero and thus simplifying the model.

\section{MATERIALS AND EXPERIMENT}

Our study area is located at the Bavarian Forest National Park, as a temperate forest located in the south eastern part of Germany. The forest dominated by Norway spruce (Picea abies) and cohabited with European beech (Fagus sylvatica) and Silver fir (Abies alba). Rare deciduous species are also present in the region such as white birch (Betula pendula), sycamore maple (Acer pseudoplatanus), common rowan (Sorbus aucuparia) European ash (Fraxinus excelsior) and European aspen (Populus tremula). 
In this experiment the $3 \mathrm{D}$ point clouds is available as a simulated MLS sensor by combining two different Lidar sensors providing three different wavelengths. The available MLS data were acquired in the summer 2016 at the same date in a leaf-on condition with an average point density of 37 point $/ \mathrm{m}^{2}$ using 3 different Riegl scanners, LMS-680i, LMS-Q780 and VQ-880-G. The combined 3D point clouds is consisting of three different spectral channels (1550 $\mathrm{nm}$ (channel 1), $1064 \mathrm{~nm}$ (channel 2) and 532 $\mathrm{nm}$ (channel 3)). The so-called intensity which is equivalent to the reflectance for the LMS-Q780 and VQ-880-G scanners, is calculated by the Riegl RiANALYZE software $\left.{ }^{(}\right)$and corrected with respect to the distance. In the case of LMS-680i scanner, the amplitude was approximately converted to the reflectance using reference amplitude values captured during the calibration flight over an airfield and by neglecting the scan angle. Fig. 1 shows an example $3 \mathrm{D}$ tree cluster with the acquired 3D point clouds.

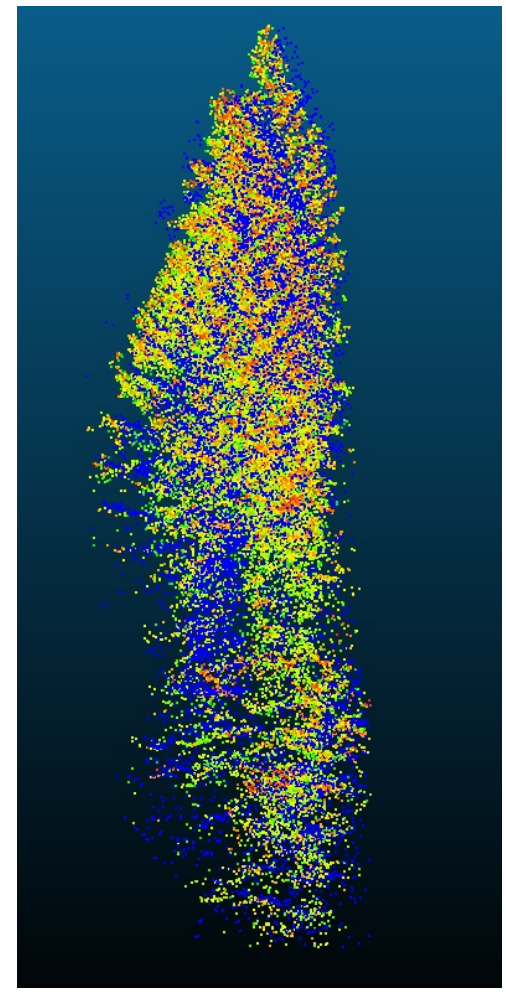

Figure 1. Visualization of an example tree cluster with MLS data. The combined 3D point clouds in the current scene are representing all three spectral channels. Red stands for channel 1 , green as channel 2 and blue for channel 3 .

Experiments were conducted using 586 ground measured single trees from 20 sample plots. Based on the available ground truth data, and due to lack of reference data for some rare species, we selected following four classes of different tree species: Norway spruce, European beech, European silver fir and Snags (standing dead trees with crown). The reference data are unbalanced and dominated by beech and spruce trees. Therefore, we performed a balancing on the input data for the classifier and ran the experiment 15 times, randomly selecting subset of the dominant class which is representative each time. Due to the better coverage of single trees on upper canopy layer by MLS data, we considered only the tree crowns which are visible from top and can be identified in the reference data via a matching strategy. We used 15fold cross validation to obtain the overall classification accuracy for the quality measure of this study as a trade-off between com- putational efficiency and reducing effects of randomness.

\section{RESULTS AND DISCUSSION}

The accuracy when adding different feature combinations are presented in Table 1.

\begin{tabular}{cc}
\hline Feature sets & Ave. overall accuracy \\
\hline \hline MLS point clouds: Radiometric & 0.72 \\
\hline MLS point clouds: Geometric & 0.68 \\
\hline $\begin{array}{c}\text { MLS point clouds: Radiometric } \\
\text { + Geometric }\end{array}$ & 0.73 \\
\hline MLS point clouds: BoW $(0.2 \mathrm{~m})$ & 0.49 \\
\hline MLS point clouds: BoW $(0.3-0.8 \mathrm{~m})$ & 0.63 \\
\hline MLS point clouds: BoW $(0.8-1.6 \mathrm{~m})$ & 0.52 \\
\hline $\begin{array}{c}\text { MLS point clouds: BoW }(0.2 \mathrm{~m}) \\
\text { + Radiometric + Geometric }\end{array}$ & 0.59 \\
\hline $\begin{array}{c}\text { MLS point clouds: BoW (0.3-0.8m) } \\
\text { + Radiometric + Geometric }\end{array}$ & 0.75 \\
\hline $\begin{array}{c}\text { MLS point clouds: BoW (0.8-1.6m) } \\
\text { + Radiometric + Geometric }\end{array}$ & 0.64 \\
\hline $\begin{array}{c}\text { Single wavelength (1550 nm): BoW (0.3-0.8m) } \\
\text { + Radiometric + Geometric }\end{array}$ & 0.69 \\
\hline $\begin{array}{c}\text { Single wavelength (1064 nm): BoW }(0.3-0.8 \mathrm{~m}) \\
\text { + Radiometric + Geometric }\end{array}$ & 0.71 \\
\hline $\begin{array}{c}\text { Single wavelength (532 nm): BoW }(0.3-0.8 \mathrm{~m}) \\
\text { + Radiometric + Geometric }\end{array}$ & 0.65 \\
\hline \hline
\end{tabular}

Table 1. Results of tree species classification.

The numbers refer to the averaged classification accuracies, whereby the best result of 0.75 is achieved by the combination of all features. The results indicate that different overall accuracies of 0.50 and 0.69 can be achieved respectively for channel 1 and channel 3. In case of single ALS wavelength as initial approach, the classification accuracy obtained only using one channel features can be improved by 10-4 through introducing various intensity based features. However, the third spectral channel $(532 \mathrm{~nm})$ features did not have any significant effect. For three spectral channels the best performance is achieved with a combination of external geometry (paraboloid fitted to crown), radiometric (channel 2 intensity histogram feature) and BoW features (spherical neighborhood size of $0.3-0.8 \mathrm{~m}$ ).

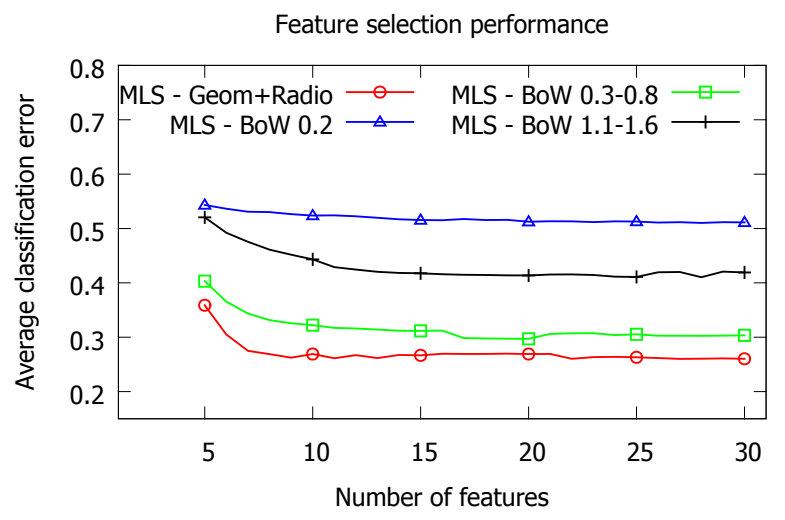

Figure 2. Learning curves describe the average error as a function of feature count; here $M L S$ refers to multispectral point clouds.

The learning curves in Fig.2 depict that about 10 features for the Bag-of-Words model (BoW) and 15 features for the rest of sets 
play a significant role in improving the accuracy. Afterwards, including more features has no benefit for the classification accuracy. Among the Bag-of-words model features, the point neighborhood of $0.2 \mathrm{~m}$ is not informative. The best results are obtained for the neighborhood size of 0.3-0.8 $\mathrm{m}$. The geometric and radiometric features outperformed BoW by nearly 9 pp, however the Bag-of-words features apparently contained additional information as their inclusion increased the final accuracy by $3 \mathrm{pp}$. Also, the separate feature selection step has a significant role in improving classification accuracy: the performance achieved with all generated features (without pre-selection) is reduced by nearly 4 pp. Fig. 3 shows a forest scene with deciduous and coniferous tree species discriminated based on intensity values in the MLS point clouds.

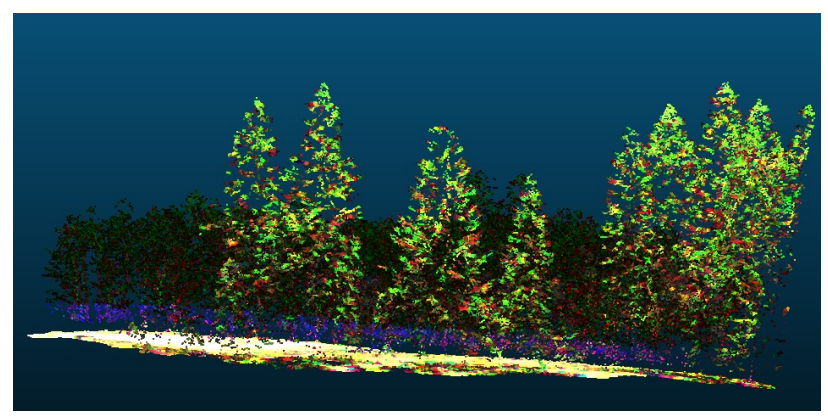

Figure 3. Visualization of an example forest area with deciduous and coniferous tree species in the MLS point clouds. Deciduous and coniferous tree species based on their intensity values are represented with different colors.

\section{CONCLUSIONS}

We have demonstrated how the various feature sets can be derived at a single tree level from MLS point clouds for tree species classification. The combination of point cloud features from three spectral channels with a pre-selection step leads to a significant improvement in the classification rate, attaining an overall accuracy of 0.75 , compared to the single wavelength approach. Our study results clearly indicate that the use of MLS data has great potential to improve the current stage of detailed forest species mapping.

\section{REFERENCES}

Fassnacht, F. E., Latifi, H., Stereńczak, K., Modzelewska, A., Lefsky, M., Waser, L. T., Straub, C. and Ghosh, A., 2016. Review of studies on tree species classification from remotely sensed data. Remote Sensing of Environment.

Ghosh, A., Fassnacht, F. E., Joshi, P. and Koch, B., 2014. A framework for mapping tree species combining hyperspectral and lidar data: Role of selected classifiers and sensor across three spatial scales. International Journal of Applied Earth Observation and Geoinformation 26, pp. 49-63.

Hastie, T., Tibshirani, R. and Friedman, J., 2001. The Elements of Statistical Learning. Springer. pp. 57-60.
Holmgren, J., Persson, Å. and Söderman, U., 2008. Species identification of individual trees by combining high resolution lidar data with multi-spectral images. International Journal of Remote Sensing.

Hovi, A., Korhonen, L., Vauhkonen, J. and Korpela, I., 2016. Lidar waveform features for tree species classification and their sensitivity to tree- and acquisition related parameters. Remote Sensing of Environment.

Jakubowski, M. K., Li, W., Guo, Q. and Kelly, M., 2013. Delineating individual trees from lidar data: A comparison of vectorand raster-based segmentation approaches. Remote Sensing 5(9), pp. 4163-4186.

Jones, T. G., Coops, N. C. and Sharma, T., 2010. Assessing the utility of airborne hyperspectral and lidar data for species distribution mapping in the coastal pacific northwest, canada. Remote Sensing of Environment 114(12), pp. 2841-2852.

Lindberg, E., Briese, C., Doneus, M., Hollaus, M., Schroiff, A. and Pfeifer, N., 2015. Multi-wavelength airborne laser scanning for characterization of tree species. Proceedings of SilviLaser pp. 271-273.

Packalén, P., Suvanto, A. and Maltamo, M., 2009. A two stage method to estimate species-specific growing stock. Photogrammetric engineering \& remote sensing 75(12), pp. 1451-1460.

Puttonen, E., Suomalainen, J., Hakala, T., Räikkönen, E., Kaartinen, H., Kaasalainen, S. and Litkey, P., 2010. Tree species classification from fused active hyperspectral reflectance and lidar measurements. Forest Ecology and Management 260(10), pp. $1843-1852$.

Reitberger, J., Schnörr, C., Krzystek, P. and Stilla, U., 2009. 3D segmentation of single trees exploiting full waveform lidar data. ISPRS Journal of Photogrammetry and Remote Sensing.

St-Onge, B. and Budei, B., 2015. Individual tree species identification using the multispectral return intensities of the optech titan lidar system. Proceedings of SilviLaser pp. 71-73.

Waser, L. T., Ginzler, C., Kuechler, M., Baltsavias, E. and Hurni, L., 2011. Semi-automatic classification of tree species in different forest ecosystems by spectral and geometric variables derived from airborne digital sensor (ads40) and rc30 data. Remote Sensing of Environment 115(1), pp. 76-85.

Waser, L. T., Klonus, S., Ehlers, M., Küchler, M. and Jung, A., 2010. Potential of digital sensors for land cover and tree species classifications-a case study in the framework of the dgpf-project. Photogrammetrie-Fernerkundung-Geoinformation 2010(2), pp. 141-156.

Weinmann, M., Jutzi, B. and Mallet, C., 2013. Feature relevance assessment for the semantic interpretation of $3 \mathrm{~d}$ point cloud data. ISPRS Annals of Photogrammetry, Remote Sensing and Spatial Information Sciences.

Yao, W., Krzystek, P. and Heurich, M., 2012. Tree species classification and estimation of stem volume and dbh based on single tree extraction by exploiting airborne full-waveform lidar data. Remote Sensing of Environment.

Yu, X., Hyyppä, J., Litkey, P., Kaartinen, H., Vastaranta, M. and Holopainen, M., 2017. Single-sensor solution to tree species classification using multispectral airborne laser scanning. Remote Sensing 9(2), pp. 108. 